\title{
Analitical definition of the law of change in the traction resistance of the plowshare and justifi- cation of its model
}

\author{
V. V. Tsybulevsky*, S. G. Rudnev, A. A. Poluektov \\ Kuban State Agrarian University, 13 Kalinina str., Krasnodar, 350044, Russia
}

\begin{abstract}
An analytical definition of the law of change in the traction resistance of the plowshare is proposed, and its mathematical model is justified. To confirm these theoretical studies, experiments were conducted in the soil channel of the North-Caucasus Research Institute of Horticulture and Viticulture. According to a specially developed program, solutions in numerical form were carried out on a PC. To solve this problem, we used the Fourier, Sturm-Liouville equations, transformed from the polar system to the Cartesian system. The results obtained for the first eight harmonics were processed by the spline-approximation program, and then a regression analysis was performed. The pair correlation coefficient was calculated. Based on the obtained values, a graph of the dependence of the soil layer's movement in the transverse plane on the forward movement was constructed.
\end{abstract}

Plowing in agriculture occupies a special place not only in terms of agrotechnical requirements, but also in terms of its energy intensity: it consumes about $30 . .50 \%$ of all energy expenditure in field production. Therefore, strict agrotechnical requirements are imposed on plowing. Currently, when using general-purpose plowshares, only $20 \%$ of the field surface meets the agrotechnical requirements of the soil layer crumbling. As a result of experiments, it was revealed that the degree of soil layer crumbling by plowshares is only 35 ... $60 \%$. Since plowing is the most energy-intensive operation, it is often replaced by other technological operations - cultivation, disking, etc. Consequently, there is no proper loosening to the depth of the arable horizon, which contributes to the re-compaction of the lower soil layers, as a result of which the water-air nutrition regime of the root layer of plants is disrupted.

As a result of repeated passage of heavy equipment on the surface of the field, it is overcompacted and degraded. It was found experimentally that when the density changes by $0.1 \ldots 0.2 \mathrm{~g} / \mathrm{cm}^{2}$ from the corresponding agrotechnical requirements, the yield of agricultural crops decreases by 15 ... 35\%.

To solve the previously obtained equation of motion of a planter unit with a free hitch [1], the generalized force $\mathrm{Q}$ was determined:

$$
Q=P_{T} \cos \beta-P_{K}-f P_{n}-P_{q x} \sin \alpha
$$

\footnotetext{
*Corresponding author: valera-1913@mail.ru
} 
where $P_{T}$ - traction force;

$P_{K}$ - reaction resulting from the action of resistance forces on the field wheel;

$P_{n}$ - soil reaction resulting from the action of the field board on the furrow wall;

$P_{q x}$ - resultant of the resistance forces in the HOY plane resulting from the soil layer pressure;

$f$ - coefficient of soil friction.

To determine the components of the right-hand side of equation (1), an experiment was prepared and conducted to determine the energy consumption according to the given parameters. However, the force depends on parameters of both the working body and the raised soil layer. Therefore, the task was set to create an equation of the movement of the soil layer on the surface of the dump in order to identify the relationship of parameters of the soil layer with the geometry of the working body [2].

To solve the problem, the equation of the soil layer motion on the surface of the dump in the HOY plane is drawn up, since, according to the work of M. L. Gusyatsky [3], the fluctuation of the processing depth of a free-radial hitch and one working body is minimal, i.e. Figure 1 shows a diagram of the action of forces that allows us to determine the nature of the movement of the soil layer on the surface of the plowshare body.

If the plowshare body is a plowing-tilling surface, then the soil layer will move along the cycloid in the HOY plane [4,5].

To solve this problem, we apply the following notation:

$\beta$ - angle of inclination of the tangent from the point of origin of the trajectory $\mathrm{C}$,

$\Psi$ - application angle,

$\rho$-density of soil layer processing,

a - plowing depth,

$\mathrm{b}$ - gripping width of a plowing unit.

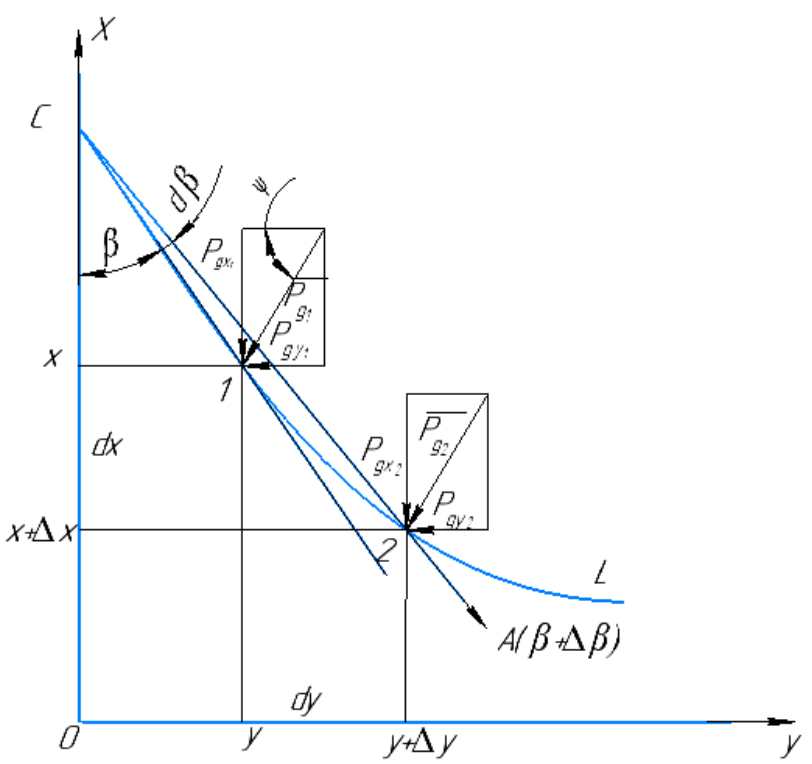

Fig. 1Diagram of the forces acting on the plowshare body. 


$$
L=\int_{0}^{\alpha} \sqrt{r^{2}+\sigma^{2}-2 r \sigma \cos \alpha} d \alpha
$$

where $\quad \mathrm{r}$ - radius of the cycloid;

$\sigma$ - cycloid compression ratio;

$\alpha$ - angle at which the soil layer is turned.

The elementary segment of the arc $\mathrm{dL}$ is shown by the formula:

$$
d L=\sqrt{d x^{2}+d y^{2}}
$$

forces moving the reservoir from point 1 to point 2 will represent the following equation (Fig.1):

$$
\sum x_{2}=-A(\beta+\Delta \beta) \cos (\beta+\Delta \beta)+A(\beta+\Delta \beta) \frac{\sin (\beta+\Delta \beta)}{\cos (\beta+\Delta \beta)}
$$

After the corresponding transformations and differentiation, the equation will take the following form:

$$
\sum x_{2}=-\frac{A \beta}{\cos \beta} d \beta
$$

Let's make the equation of the reservoir motion in the X-plane.

The mass of the transported reservoir will be equal to:

$$
m=\rho a b d x
$$

After transformation

$$
(\rho a b d x) x_{1}=-\frac{A \beta}{\cos \beta} d \beta
$$

knowing that

$$
\frac{A \beta}{\cos \beta} d \beta=P_{q x}
$$

and

$$
(\rho a b d x) x_{1}=-P_{q x} d \beta
$$

Dividing the left and right parts of expression (8) by $\mathrm{d} ß$, we get:

$$
\rho a b \frac{d^{2} x}{d t^{2}}=-P_{q x} \frac{d \beta}{d x}
$$

Similarly, we make the equation of motion of the reservoir relative to the Y-axis:

$$
\rho a b \frac{d^{2} y}{d t^{2}}=P_{q x}(\cos \beta \sin \beta-\operatorname{ctg} x) \frac{d \beta}{d x}
$$

The acceleration of the movement of the soil layer along the trajectory $\mathrm{L}$ will have the form:

$$
\frac{d^{2} L}{d t^{2}}=\sqrt{\frac{d^{2} x}{d t^{2}}-\frac{d^{2} y}{d t^{2}}}
$$


By differentiating the expression (2) twice, we find the acceleration of the motion along the trajectory $\mathrm{Lj}$

$$
\frac{d^{2} S}{d t^{2}}=2 r \cos \frac{\alpha}{2} \frac{d^{2} \alpha}{d t^{2}}
$$

After substituting expressions (9),(10),(12) in the expression (11) and after performing the necessary transformations, we find the equation of the soil layer motion on the surface of the plowshare body:

$$
\frac{d^{2} y}{d t^{2}}=\frac{P_{q x}}{\rho a b} \sqrt{1+(\cos \beta \sin \beta-\operatorname{ctg} x)^{2}} \cos ^{2} \beta \cos \varphi \frac{d^{2} y}{d x^{2}}
$$

As a result of the transformation, a second-order partial differential equation was obtained.

Under known initial conditions,

$$
y(x, 0)=f(x) ; \frac{d y(x, 0)}{d t}=A(x)
$$

these conditions show where the soil layer is located at the initial time and the velocity of its point at $\mathrm{t}=0$, where $\mathrm{f}(\mathrm{x})$ and $\mathrm{A}(\mathrm{x})$ are known functions and variables.

Border conditions show that the soil layer moves in a horizontal plane.

$$
y(0, t)=y(k, t)=0
$$

Using the Fourier method, we solve the auxiliary problem.

$$
y(x, t) \neq 0
$$

or equation

$$
y(x, t)=Q(x) N(t)
$$

which fits the specified conditions

$$
\begin{aligned}
& Q(o) N(t)=0 \\
& Q(l) N(t)=0 \text { для всех } t>0
\end{aligned}
$$

After that, substituting the expression (17) in the expression (13), we get

$$
\frac{P_{q x}}{\rho a b} \sqrt{1+(\cos \beta \sin \beta-\operatorname{ctg} x)^{2}} \cos ^{2} \beta \cos \alpha=\mathrm{a}^{2}
$$

As a result of the transformation, we obtain two differential equations:

$$
\begin{aligned}
X Q^{\prime}+\gamma Q & =0 \\
N^{\prime \prime}+E^{2} \gamma N & =0
\end{aligned}
$$

As a result of solving the Sturm-Liouville problem, the coefficient $\gamma$ is found, as well as non-trivial functions:

$$
Q_{k}(x)=O \sin \tau s \quad(\tau=1,2 \ldots n)
$$

where $x=l \cos \alpha$ on problem situation (15).

$$
M_{\tau}(t)=C_{\tau} \cos \Psi k t+Z_{\tau} \sin \Psi k t(\tau=1,2 \ldots n)
$$

Hence, the general solution of equation (13) will be: 


$$
y(x, t)=\sum_{\tau=1}^{n}\left(\mathrm{C}_{\tau} \cos \Psi \tau t+B_{\tau} \sin \Psi \tau t\right) \sin \tau_{x}
$$

After solving this expression, the equation of harmonic oscillations that are formed during the rise of the soil layer was obtained [6]. Since the vibrations of the layers are the sum of free and forced vibrations, the equation will take the following form.

$$
y=j(x)+i(x)
$$

where $j(x)$-forced vibrations generated by the driving force,

$i(x)$ - free fluctuations..

The soil layer in the horizontal plane moves along the cycloid, then

$$
f(x)=r \sqrt{2(1-\cos \alpha)}
$$

Converting (26) from the polar system to the Cartesian coordinate system, substituting (24) and (26) into (25) and after a series of transformations we get:

$$
(x)=2\left(\frac{P_{q x}}{a n \pi} \int_{0}^{b} \sin ^{2} \frac{a n \pi}{l} \sin \frac{\pi x}{l} d x+\frac{1}{l} \int_{0}^{l}-\frac{x}{\sqrt{32 r^{2}-x^{2}}} \sin \frac{\pi x}{l} d \sin \frac{a n \pi}{l}\right) \sin \frac{\pi x}{l}
$$

where

$$
a=\sqrt{\frac{P_{q x}}{\rho a b} \sqrt{1+(\cos \beta \sin \beta-\operatorname{ctg} \Psi)^{2}} \cos ^{2} \beta \cos \alpha}
$$

Expression (27) is a partial solution of equation (13), which has been decomposed into the Fourier series. Knowing that the function $f(x)$ is odd, that is, $f(x) \neq f(-x)$.

Since the complete solution of expression (13) is very cumbersome, it is performed in numerical form on a PC using a specially compiled program [7]. The resulting series of paired dependencies $\mathrm{y}$ and $\mathrm{x}$ for the first 8 harmonics was then processed on a PC by specially developed spline-approximation programs and then a regression analysis was performed. The 16 dependencies were analyzed on a PC. As a result of the experiment, the regression equation was found:

$$
y=\frac{x}{0,0272 x-0,0904}
$$

After further analysis, the formula was obtained for finding the pair correlation coefficient:

$$
R=\frac{\frac{\sum_{i=1}^{n} x_{i} y_{i}-\left(\sum_{i=1}^{n} x_{i} \sum_{i=1}^{n} y_{i}\right)}{n}}{\sqrt{\sum_{i=1}^{n} x_{i}^{2}-\frac{\left(\sum_{i=1}^{n} x_{1}\right)^{2}}{n}}-\sqrt{\sum_{i=1}^{n} y_{i}^{2}-\frac{\left(\sum_{i=1}^{n} y_{1}\right)^{2}}{n}}}
$$

Its value was determined after calculations on the PC numerically:

$$
R=-0,925207
$$

Based on the obtained values, a graph was constructed (Fig.2), showing the dependence of the movement of the soil layer in the transverse plane on the translational motion.

The shaded section of the curve shows the effect of 5-8 harmonics of forced oscillations. Therefore, we can draw the following conclusion: reducing the traction resistance of the plowshare body is possible by reducing the number of higher harmonic components, which can be provided by choosing the optimal design of the tilled surface and the associated rational trajectory of the soil layer. 
To determine the numerical values of the force parameters in equation (1), the experiment was prepared and conducted in the soil channel of the South-Caucasus Research Institute of Horticulture and Viticulture. The channel length of $15 \mathrm{~m}$ and width of $1.2 \mathrm{~m}$ did not allow testing the working body in full size, so a model of a planter with a free hitch was developed.

The following parameters were taken into account in the simulation [8 ... 10].

D -geometric dimensions of the plowshare body;

$\mathrm{V}$-speed of the forward movement of the arable unit;

$\rho$ - density of the soil layer;

$\mathrm{S}$ - soil layer cross-sectional area;

Let's define the scale indicator using the following formula:

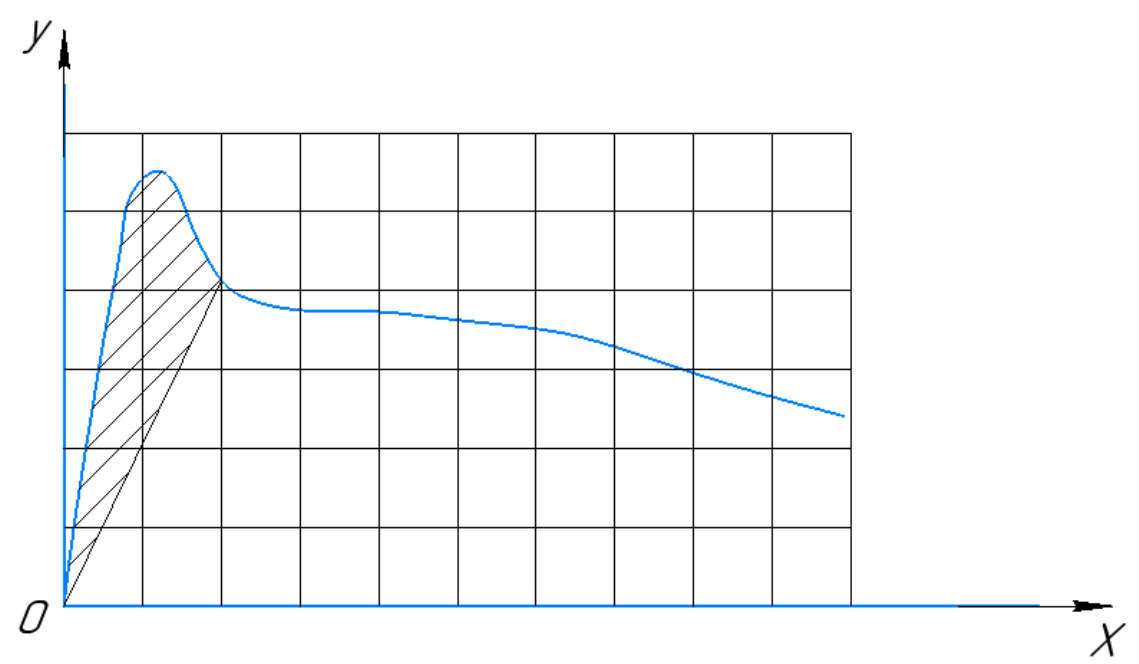

Fig. 2. Dependence $y=f(x)$.

$$
g=\frac{u_{\Pi}}{u_{\mathrm{M}}}
$$

where $u_{\Pi}$ - spatial dimensions of a natural plowshare;

$u_{\mathrm{m}}$ - spatial dimensions of the model.

Then, according to the theory of similarity and dimensions, if we take the ratio of soil densities:

$$
\frac{\rho_{\mathrm{H}}}{\rho_{\mathrm{M}}}=\eta
$$

we can write the following expression for the model:

Speed:

$$
V_{\mathrm{M}}=\frac{V_{\mathrm{H}}}{\sqrt{g}}
$$

soil layer cross-sectional area:

$$
S_{\mathrm{M}}=\frac{S_{\mathrm{H}}}{g^{2}}
$$

weight of the working body (unit): 


$$
m_{\mathrm{M}}=\frac{m_{\mathrm{H}}}{s g^{3}}
$$

traction resistance:

$$
M=\frac{R}{\beta l^{2} V^{2}}
$$

or with regard to (33):

$$
R_{\mathrm{M}}=\frac{R_{\mathrm{H}}}{s g^{3}}
$$

The above similarity criteria allowed us to create a device with parameters close to the real unit, and as a result of the tests to determine the optimal geometry of both the plowshare and the hitching units.

\section{References}

1. Peisakhovich Yu.A. To substantiation of a model of an improved planter with a free hitch [Text]/ A.Yu. Peisakhovich // Improving the use and maintenance of the machine and tractor fleet. Thematic proceedings / KSAI. Krasnodar 1987. Issue 272 (300).

2. Drobot V.A., Tsybulevsky V.V. Optimization of parameters of the surface tillage process of a horizontally positioned disk working body. [Text] / V. V. Drobot, V.V. Tsybulevsky // Proceedings of Kuban State Agrarian University. 2010. № 23. P.181-186.

3. Gusyatsky M.L. // Fundamentals of the theory of wheel units [Text] /.M.L. Gusyatsky // Proceedings of VIM. Vol. 46. M. 1970.

4. Vygotsky M.Ya. // Handbook of Higher Mathematics [Text] / M.Ya. Vygotsky // M. Nauka. 1973.

5. MULTILAYERED DESTRUCTION OF A SOIL LAYER / Petunina I.A., Rudnev S.G. - Proceedings: IOP Conference Series: Earth and Environmental Science. Russian Conference on Technological Solutions and Instrumentation for Agribusiness, TSIA 2019.2020. P. 012038.

6. Gainanov Kh.S. Application of the theory of similarity and dimension for modeling the working bodies of tillage units [Text] / Kh.S. Gainanov // Proceedings of Kazan Agricultural Institute. 1970. Issue 55.

7. Dyakonov V.P., Abramenkova I.V. MathCAD 7.0 in Mathematics, Physics, and in Internet [Text] / V. P. Dyakonov, I. V. Abramenkova. - M.: «Nolidg», 1999. - 352 p., illustrated.

8. OPTIMIZATION OF PARAMETERS OF ECOLOGICAL-MELIORATIVE PRODUCT FOR PRE-SEED TREATMENT OF SOIL / Tarasenko B.F., Oskin S.V., Tsybulevsky V.V., Karpenko V.D., Rudnev S.G.: IOP Conference Series: Earth and Environmental Science. Russian Conference on Technological Solutions and Instrumentation for Agribusiness, TSIA 2019.2020. P. 012045.

9. Adler, V. A. Introduction into planning of an experiment [Text] / V. A. Adler. - M.: Metallurgy, 1999. - 159 p.

10. Novik, F. S., Arsov, A. B. Optimization of metal technology processes by experimental planning methods [Text] / F. S. Novik, A. B. Arsov. - M.: Mechanical engineering, 1980. 